\title{
Toll-like receptors 7 and 8 expression correlates with the expression of immune biomarkers and positively predicts the clinical outcome of patients with melanoma
}

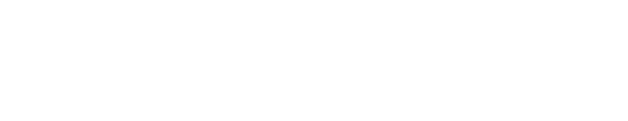

\section{Moucheng Zhang ${ }^{1-3}$ \\ Zhilong Yan ${ }^{2}$ \\ Junjiang Wang ${ }^{1,3}$ \\ Xueqing Yao ${ }^{1,3}$}

'Second Clinical Medical College of Southern Medical University, Guangzhou, ${ }^{2}$ Department of Gastrointestinal Surgery, First Hospital of Ningbo, Ningbo, Zhejiang, ${ }^{3}$ Department of General Surgery, Guangdong General Hospital and Guangdong Academy of Medical Sciences, Guangzhou, Guangdong, China
Correspondence: Xueqing Yao Department of General Surgery, Guangdong General Hospital and Guangdong Academy of Medical Sciences, 106 Zhongshan 2nd Road, Guangzhou, Guangdong 510080, China

Tel +86 I390238 8635

Email yjb92I I@2Icn.com
Background: Toll-like receptors (TLRs) play a critical role in cancer, yet the clinical relevance of TLR7/8 expression in melanoma remains unclear. This study aimed to evaluate the prognostic value of TLR7/8 mRNA levels in melanoma and their correlation with immune biomarkers relevant to disease progression.

Methods: Normalized gene expression and corresponding clinical data of patients with skin cutaneous melanoma were obtained from two public databases: the Cancer Genome Atlas and GSE19234. Log rank (Mantel-Cox) tests were used to perform survival analysis. Multivariate survival analysis was performed on a Cox-regression hazard model. Spearman correlation analyses were used to address the relationship between the expressions of TLR7/8 levels and immune biomarkers in melanoma tumors.

Results: Survival analysis suggested that high levels of TLR7 or TLR8 expression predicted better clinical outcome for melanoma patients (TLR7: HR =1.734, $P<0.0001$; TLR8: HR =2.072, $P<0.0001)$. Moreover, multivariate survival analysis implicated TLR7 as a prognostic factor independent of age, gender, or pathological stage. Further analysis demonstrated that expression levels of TLR7/8 strongly correlated with that of dendritic cell markers and chemokines/ chemokine receptors, including CCR2, CCR5, CCL3, and CCL5. Importantly, expression levels of both TLR7 and TLR8 were also highly correlated with the expressions of CD8 and other functional markers of $\mathrm{CD} 8^{+} \mathrm{T}$ cells.

Conclusion: High gene expression of TLR7 and TLR8 in melanoma tumors is associated with high expression levels of functional markers of immune cells, which predicts longer overall survival of patients with melanoma. Our results not only provide an important reference for the clinical prognosis of melanoma but also present new implications for the design of melanoma immunotherapy.

Keywords: TLR7, TLR8, melanoma, prognosis, survival, immune marker, immunotherapy

\section{Introduction}

Toll-like receptors (TLRs) are signaling molecules that recognize pathogen-associated molecular patterns derived from bacteria, virus, or fungi. ${ }^{1}$ Activation of TLRs triggers several signaling pathways and regulates downstream expression of a variety of genes. TLRs are, therefore, crucial for both innate immunity and adaptive immune responses. ${ }^{2}$ Aberrant regulation of TLRs in human skin could, in part, lead to chronic inflammation and contribute to melanoma tumorigenesis. TLR7 and TLR8 (TLR7/8) are expressed in immune cells, including dendritic cells (DCs), as well 
as in keratinocytes and melanoma cells. ${ }^{3}$ TLR7 binds to single-stranded RNA (ssRNA) of viruses or bacteria. TLR8 is phylogenetically related to TLR7 and, like TLR7, binds to viral ssRNAs and foreign bacteria. ${ }^{4}$ Specific agonists of TLR7 and TLR 8 have been used as targeted therapies in a variety of cancers, including pancreatic and bladder cancers, basal cell carcinoma, and melanoma..$^{1,3,5-7}$

Activation of TLRs using agonists can modulate the signaling pathways of MyD88/NF-kB and interferon (IFN) regulatory factors (IRF) $3 / 7$. TLR7/8 activation may thus result in the upregulation of chemokines and their receptors, either positively or negatively affecting tumor infiltration of immune cells. ${ }^{8,9}$ Although TLR7 agonists have been used to treat cancer patients, their ability to generate tumor-specific immunity and to kill tumor cells directly by activating innate immune responses remains controversial. ${ }^{10,11}$ Moreover, it has been theorized that TLR7 agonists may provoke inflammation and promote tumorigenesis in some cancer types. ${ }^{12}$ Though few studies have addressed the clinical relevance of TLR7/8 in terms of its prognostic value in cancer and its association with immunological biomarkers, it is imperative to address the precise role of TLR7/8 during cancer progression.

In this study, we performed a systematic analysis of tumor TLR7/8 expression levels and survival data from a large cohort of melanoma patients provided by the publicly available databases, The Cancer Genome Atlas (TCGA) and GSE19234. Our data highlight the prognostic value of TLR7/8 in melanoma and we address its association with the expression of immune cell markers.

\section{Methods}

\section{Skin cutaneous melanoma database query and processing}

The calculated and normalized gene expression (level 3) data, and corresponding clinical data, of 473 melanoma samples were acquired from TCGA through the UCSC cancer browser. ${ }^{13}$ We excluded duplicate samples from same patients and samples from patients without available survival data, with a final cohort of 427 patients for survival analysis. The clinical information of skin cutaneous melanoma (SKCM) patients used in this study included age, gender, and pathological TNM (tumor, node, and metastases) stage. Among the cohort of 427 patients, there were $\sim 10 \%$ of patients who received radiation therapy (21/214 for TLR7 high vs 20/213 for TLR7 low) prior to the sample collection. For GSE19234 database sampling, ${ }^{14}$ gene expression and clinical data were downloaded from the Gene Expression Omnibus (GEO) and processed using the R package "GEOquery".

\section{Survival analysis}

To evaluate the prognostic value of TLR7/8, we categorized the patients into two groups, high and low, based on the median of TLR7 or TLR8 gene expression, respectively. Survival analysis between the "high" group and the "low" group in each analysis was assessed by Kaplan-Meier survival analysis and compared by two-sided log-rank test. To determine whether the prognostic value of TLR7/8 was independent of other clinical variables (eg, age, gender, and tumor stage), multivariate Cox regression was performed on each dataset by using the R package "survival", with survival as the dependent variable and TLR7 expression and other clinical variables as explanatory variables. Hazard ratios (HR) and 95\% confidence intervals (CI) were calculated.

\section{Correlation analysis}

Pearson's correlation tests were used to study the association between the expressions of TLR7/8 and genes of interest in tumor samples. HRs and 95\% CIs were then calculated. All graphical presentations were done using GraphPad Prism 6.0.

\section{Results}

\section{High levels of TLR7/8 expression predict better prognoses in patients with melanoma}

Taking advantage of the publicly available database TCGA, we accessed the normalized tumor gene expression levels and corresponding clinical data of 473 SKCM patients. As shown in Figure 1A, our survival analysis indicated that high TLR7 expression levels predict longer overall survival times (HR $=1.734, P=0.0002$ ) for these patients. Similar results were observed in the survival analysis stratified with TLR8 expression levels (HR $=2.072, P<0.0001$, Figure 1B). To validate these results, we analyzed a second dataset, GSE $19234,{ }^{14}$ with gene expression levels and clinical data of 44 melanoma patients. Despite the smaller patient cohort, our survival analysis with the GSE19234 database indicated that high levels of TLR7 expression also predict longer overall survival times $(\mathrm{HR}=2.492, P=0.023$, Figure $1 \mathrm{C})$. A similar trend was observed in our survival analysis stratified with TLR8 expression levels (HR $=1.772, P=0.19$, Figure 1D).

Both TLR7 and TLR 8 genes are located in human chromosome $\mathrm{Xp} 22.2 .{ }^{4} \mathrm{We}$, therefore, sought to assess the association between TLR7 and TLR8 expression levels. Our results indicated a strong correlation between the expression levels of TLR7 and TLR8 ( $r=0.8903, P<0.0001$, Table 1$)$. Next, we performed multivariate survival analysis and found 

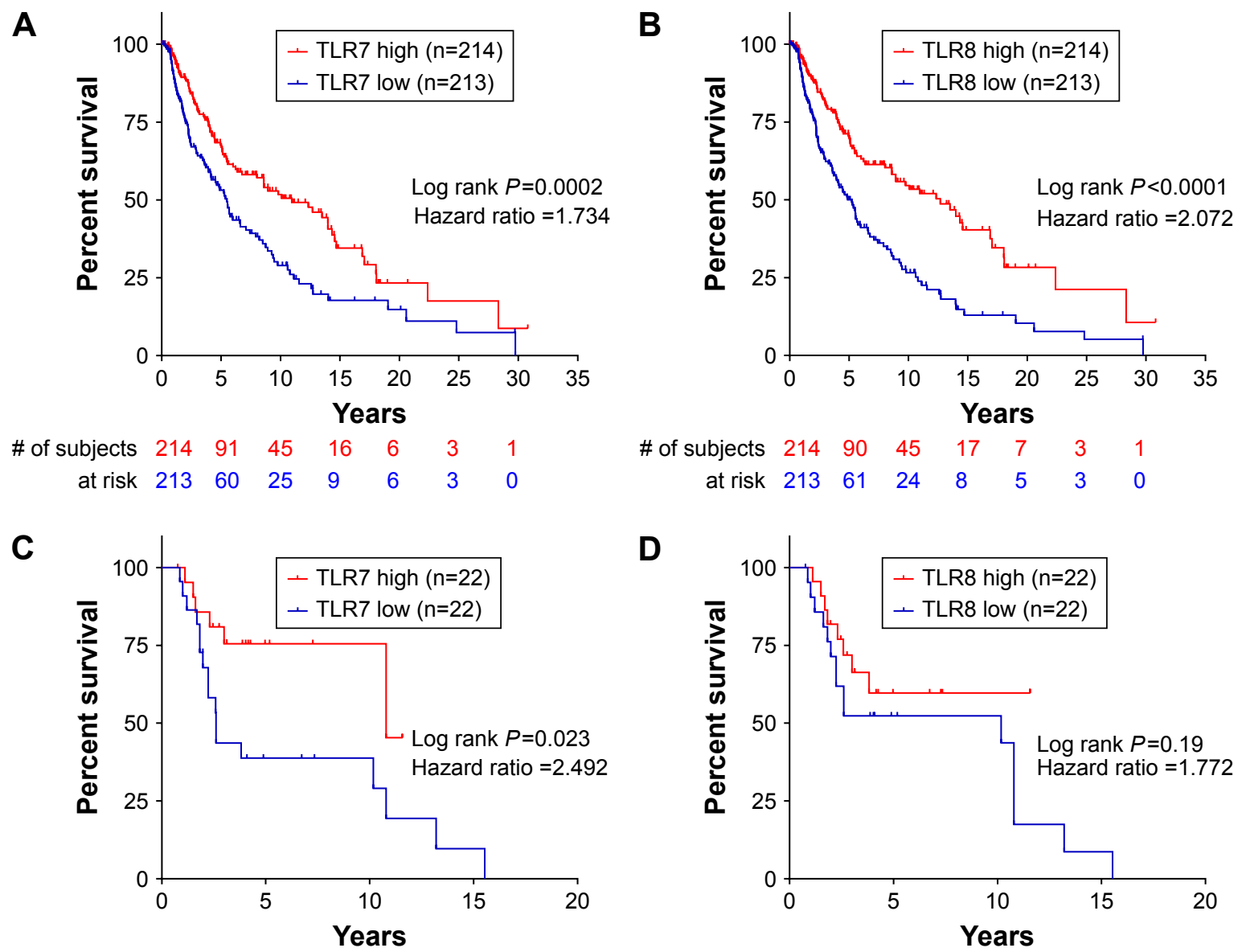

Figure I Transcriptional levels of TLR7 and TLR8 predict the clinical outcome of patients with melanoma. Survival analysis of melanoma patients (TCGA database) is shown with high and low expressions of TLR7 (A) and TLR8 (B). Survival analysis of melanoma patients (GSE 19234) is shown with high and low expressions of TLR7 (C) and TLR8 (D). The patients were divided into two groups based on the median mRNA expression levels of TLR7 and TLR8.

Abbreviations: TLR, Toll-like receptor; TCGA, The Cancer Genome Atlas.

that high TLR7 expression levels were significantly associated with better prognosis independent of other variables (Table 2). These results suggest that TLR7 is a prognostic factor of melanoma independent of age, gender, and TNM stage.

\section{TLR7/8 expression levels are correlated with the expression of CDI Ib and CD I Ic} TLR7/8 has been found to be expressed in macrophages, monocytes, and DCs. We, therefore, assessed the correlation between the expressions of TLR7/8 levels and DC-specific markers. We found a strong correlation between the expressions of TLR7 levels and CD11b (ITGAM) and CD11c (ITGAX) levels (Figure 2 and Table 1). Expression levels of TLR7 correlated well with that of FLT3G, an important marker for a subset of DCs, but not with the levels of IFN $\beta$ (IFNB1) or IRF3. These findings confirm the relationship between TLR7/8 expression and DC infiltration, though the role TLR7 plays in activating downstream signaling requires further functional analysis.

\section{Expression levels of TLR7/8 are correlated with that of chemokines and their receptors}

To test whether TLR7/8 is involved in immune cell infiltration and trafficking, we performed correlation analysis between the expression levels of TLR7/8 and chemokines and their receptors. Our data showed that the levels of TLR7 strongly correlated with that of CCR2 $(r=0.8337, P<0.0001)$ and CCR5 ( $r=0.8448, P<0.0001)$ (Figure 3 and Table 1). There was also a correlation between the expression levels of TLR7/8 and chemokine (Figure 3 and Table 1).

\section{Expression levels of TLR7/8 are correlated with that of $\mathrm{CD}^{+} \mathrm{T}$-cell \\ functional markers}

Because high levels of $\mathrm{CD} 8^{+} \mathrm{T}$-cell infiltration have been shown to be associated with better survival rates in patients with melanoma, ${ }^{15}$ we sought to investigate the correlation 
Table I Correlation analysis of TLR7/8 and genes of interest

\begin{tabular}{|c|c|c|c|c|c|c|}
\hline \multirow[t]{2}{*}{ Genes } & \multicolumn{3}{|l|}{ TLR7 } & \multicolumn{3}{|l|}{ TLR8 } \\
\hline & Pearson's r & $95 \% \mathrm{Cl}$ & $P$-value & Pearson's $r$ & $95 \% \mathrm{Cl}$ & $P$-value \\
\hline CCL2 & 0.5852 & $0.5225-0.64 I 5$ & $<0.0001$ & 0.6211 & $0.5624-0.6736$ & $<0.0001$ \\
\hline CCL3 & 0.5302 & $0.4622-0.5921$ & $<0.0001$ & 0.615 & $0.5557-0.6682$ & $<0.0001$ \\
\hline CCL4 & 0.6982 & $0.6488-0.7417$ & $<0.0001$ & 0.8052 & $0.7710-0.8348$ & $<0.0001$ \\
\hline CCL5 & 0.6795 & $0.6278-0.7252$ & $<0.0001$ & 0.7656 & $0.7255-0.8005$ & $<0.0001$ \\
\hline CCR2 & 0.8337 & $0.8039-0.8592$ & $<0.000 \mathrm{I}$ & 0.8662 & $0.84|8-0.887|$ & $<0.0001$ \\
\hline CCR5 & 0.8448 & $0.8169-0.8688$ & $<0.000 \mathrm{I}$ & 0.904 & $0.8860-0.9192$ & $<0.0001$ \\
\hline$C D 3 E$ & 0.7355 & $0.6912-0.7744$ & $<0.0001$ & 0.7949 & $0.759 \mid-0.8258$ & $<0.0001$ \\
\hline$C D 4$ & 0.8087 & $0.7750-0.8378$ & $<0.0001$ & 0.8267 & $0.7959-0.8533$ & $<0.0001$ \\
\hline CD8A & 0.7329 & $0.6882-0.7720$ & $<0.0001$ & 0.8204 & $0.7885-0.8478$ & $<0.0001$ \\
\hline$C D 8 B$ & 0.7052 & $0.6568-0.7478$ & $<0.000 \mathrm{I}$ & 0.7899 & $0.7534-0.8216$ & $<0.0001$ \\
\hline FLT3 & 0.7221 & $0.6760-0.7627$ & $<0.0001$ & 0.7331 & $0.6885-0.7723$ & $<0.0001$ \\
\hline FLT3LG & 0.5328 & $0.4649-0.5944$ & $<0.0001$ & 0.5399 & $0.4727-0.6008$ & $<0.0001$ \\
\hline GZMA & 0.7354 & $0.6911-0.7743$ & $<0.0001$ & 0.8283 & $0.7977-0.8546$ & $<0.0001$ \\
\hline GZMB & 0.5902 & $0.5282-0.6460$ & $<0.0001$ & 0.7203 & $0.6739-0.7610$ & $<0.0001$ \\
\hline GZMK & 0.7773 & $0.7390-0.8107$ & $<0.0001$ & 0.8406 & $0.8120-0.8652$ & $<0.0001$ \\
\hline IFNAI & -0.0344 & -0.1242 to 0.05591 & 0.4549 & -0.03214 & -0.1220 to 0.05821 & 0.4856 \\
\hline IFNARI & 0.3979 & $0.3192-0.4712$ & $<0.000 \mathrm{I}$ & 0.3468 & $0.2649-0.4237$ & $<0.0001$ \\
\hline IFNAR2 & 0.4473 & $0.372 I-0.5166$ & $<0.000 \mathrm{I}$ & 0.4728 & $0.3997-0.5400$ & $<0.0001$ \\
\hline IFNBI & 0.2058 & $0.1178-0.2905$ & $<0.0001$ & 0.1833 & $0.09463-0.2690$ & $<0.0001$ \\
\hline IFNG & 0.648 & $0.5925-0.6975$ & $<0.0001$ & 0.7714 & $0.7321-0.8055$ & $<0.0001$ \\
\hline IFNGRI & 0.4204 & $0.3433-0.4920$ & $<0.0001$ & 0.4327 & $0.3564-0.5032$ & $<0.0001$ \\
\hline IRF3 & 0.03714 & -0.05322 to 0.1269 & 0.4203 & 0.06945 & -0.02086 to 0.1586 & 0.1315 \\
\hline IRF7 & 0.2944 & $0.2098-0.3747$ & $<0.000 \mathrm{I}$ & 0.2596 & $0.1735-0.3418$ & $<0.0001$ \\
\hline ITGAE & 0.3322 & $0.2495-0.4101$ & $<0.0001$ & 0.3708 & $0.2904-0.446 I$ & $<0.0001$ \\
\hline ITGAM & 0.7734 & $0.7344-0.8073$ & $<0.0001$ & 0.7869 & $0.7500-0.8190$ & $<0.0001$ \\
\hline ITGAX & 0.5687 & $0.5044-0.6268$ & $<0.0001$ & 0.6178 & $0.5587-0.6706$ & $<0.0001$ \\
\hline MYD88 & 0.4415 & $0.3659-0.5113$ & $<0.0001$ & 0.4432 & $0.3678-0.5129$ & $<0.0001$ \\
\hline PRFI & 0.6673 & $0.6|4|-0.7 \mid 45$ & $<0.0001$ & 0.8007 & $0.7658-0.8309$ & $<0.0001$ \\
\hline TLR8 & 0.8903 & $0.8700-0.9076$ & $<0.0001$ & & & \\
\hline
\end{tabular}

Abbreviations: $\mathrm{Cl}$, confidence interval; TLR, Toll-like receptor.

between the expression levels of TLR7 and CD8 ${ }^{+} \mathrm{T}$-cell response-related genes. As shown in Figure 4 and Table 1, we found that TLR7 expression levels are significantly associated with CD8A expression $(r=0.7329, P<0.0001)$. More importantly, expression levels of TLR7/8 also correlated with genes such as perforin (PRF1), granzyme B (GZMB), and IFNg (IFNG), which are well-known indicators of CD8 ${ }^{+}$ T-cell cytotoxic function. These findings suggest that TLR7/8 expression is associated with $\mathrm{CD} 8^{+} \mathrm{T}$-cell tumor infiltration and functions.

Table 2 Multivariate cox analyses applied to survival model

\begin{tabular}{llll}
\hline Parameters & HR & $\mathbf{9 5 \% ~ C l ~}$ & P-value \\
\hline TLR7 (low vs high) & 1.982 & $1.463-2.687$ & $<0.000$ I \\
Gender (female vs male) & 1.046 & $0.7598-1.440$ & 0.783 \\
Age & 1.026 & $1.0159-1.037$ & $<0.000$ I \\
TNM stage (III and IV vs I and II) & 1.671 & $1.23-2.269$ & 0.001 \\
\hline
\end{tabular}

Abbreviations: $\mathrm{HR}$, hazard ratio; $\mathrm{Cl}$, confidence interval; TNM, tumor, node, and metastases; TLR, Toll-like receptor.

\section{Discussion}

TLRs are important components of innate immunity in skin tissues and their dysregulation has been shown to contribute to the development of melanoma. ${ }^{1,16}$ The role of TLRs in cancer is controversial, as increased expression and activation of these proteins may result in pro- or antitumorigenic effects depending on the cellular context. Ligand activation of TLRs on DCs triggers type-I IFN expression and contributes to killing and removing cancer cells. ${ }^{17}$ It was previously reported that elevated TLR9 expression levels are associated with better overall survival in patients with breast cancer. ${ }^{18}$ However, some studies also have reported that aberrant activation of TLR enhances inflammation and promoted tumorigenesis. ${ }^{16,17}$ Noteworthy, a recent study that immunohistologically analyzed TLR7 expression levels in a small number $(\mathrm{n}=30)$ of cutaneous malignant melanoma patients did not establish a significant correlation between TLR7 protein expression and relapse-free survival. ${ }^{12}$ Thus, it 

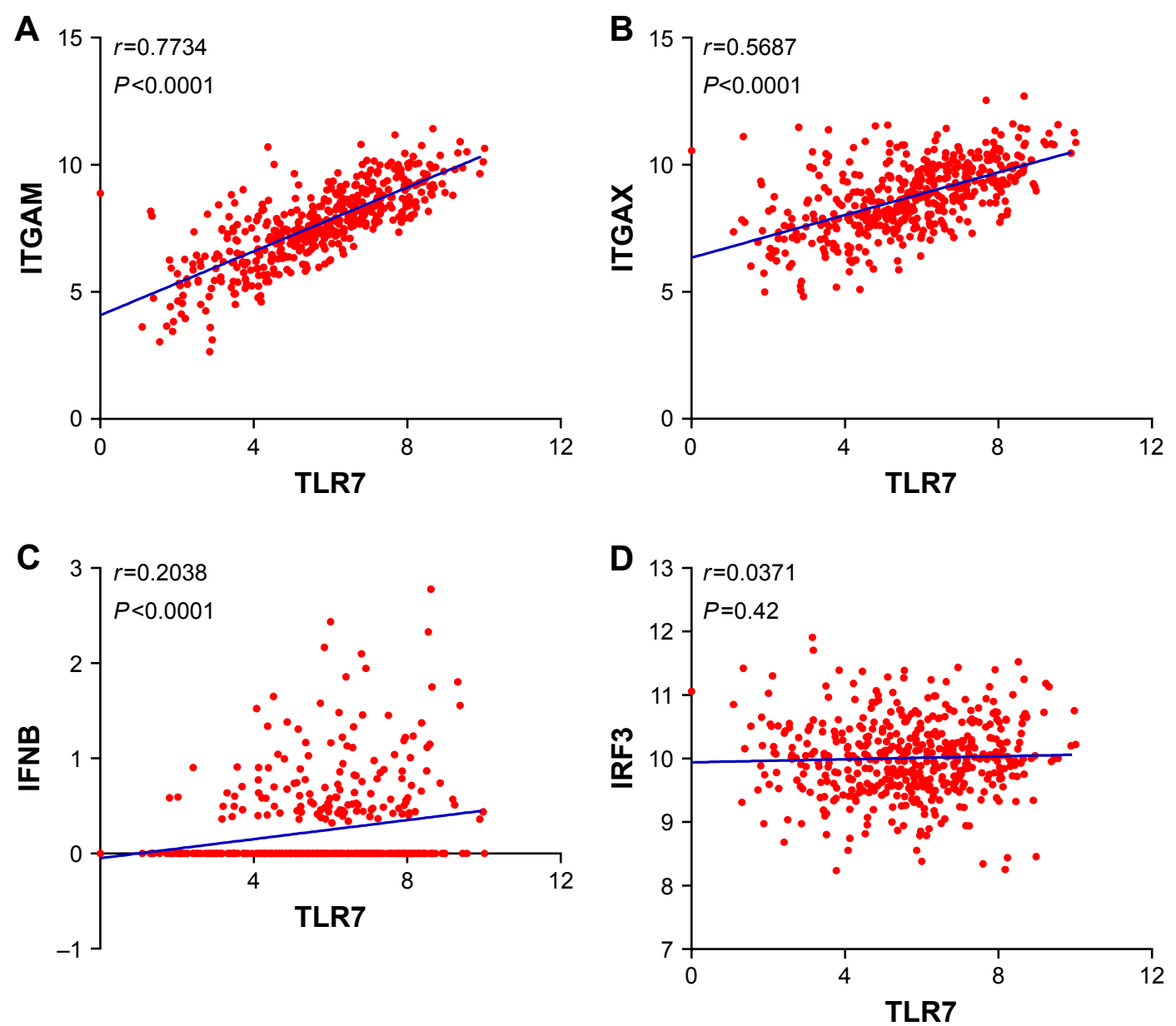

Figure 2 Correlation analysis between expressions of TLR7 and factors associated with dendritic cells (DC). Correlation analysis between (A) TLR7 and ITGAM, (B) TLR7 and ITGAX, (C) TLR7 and IFNB, and (D) TLR7 and IRF3.

Note: $r$, Pearson's correlation coefficient.

Abbreviations: TLR, Toll-like receptor; IRF3, interferon regulatory factor 3; IFNB, interferon B.

is imperative to clarify the prognostic value of TLR7 using a larger patient cohort. In our current study, we analyzed the TCGA SKCM database, with more than 400 patients' data, and revealed the clinical prognostic value of TLR7/8 in melanoma. Here, we observed that patients with high tumor expression levels of TLR7/8 had longer overall survival rates. Our results were validated using another published dataset, GSE19234. Moreover, according to multivariate analysis, high expression level of TLR7/8 protein was a prognostic factor independent of age, gender, or TNM stage. To the best of our knowledge, this is the first report to demonstrate a positive prognostic value of TLR7/8 in melanoma.

TLRs are expressed on the surface of immune cells, such as DCs, which are antigen-presenting cells. In agreement, our data show a strong correlation between the expressions of TLR7/8 and DC-specific markers, such as CD11c. The binding of endogenous ligands or synthetic agonists to TLRs triggers a cascade of signaling events and the upregulation of several chemokines and their receptors. ${ }^{16}$ Indeed, intratumoral administration of a TLR7/8 agonist generated systemic antitumor immunity against B16.F10 melanoma tumors accompanied by an increase in the levels of CCL2 and infiltration of M1 phenotype-shifted macrophages. ${ }^{19}$ TLR7/8 activation also promotes tumor eradication by activating a combination of immune cells, chemokines, and chemokine receptors at the tumor site..$^{20,21}$ In parallel, our data indicate that expression levels of TLR7/8 are strongly correlated with that of CCR2, CCR5, and their respective ligands, CCL2, CCL3, CCL4, and CCL5. We speculate that high levels of TLR7/8 in melanoma induce a chemoattracting microenvironment, ultimately resulting in the recruitment of immune cells to the tumor site.

TLR activation has been shown to promote DC maturation and the production of cytokines, potentially resulting in the activation of specific type of T-cell immunity, involving the recruitment of $\mathrm{CD}^{+} \mathrm{T}$ cells. ${ }^{22,23}$ Tumor-infiltrating 

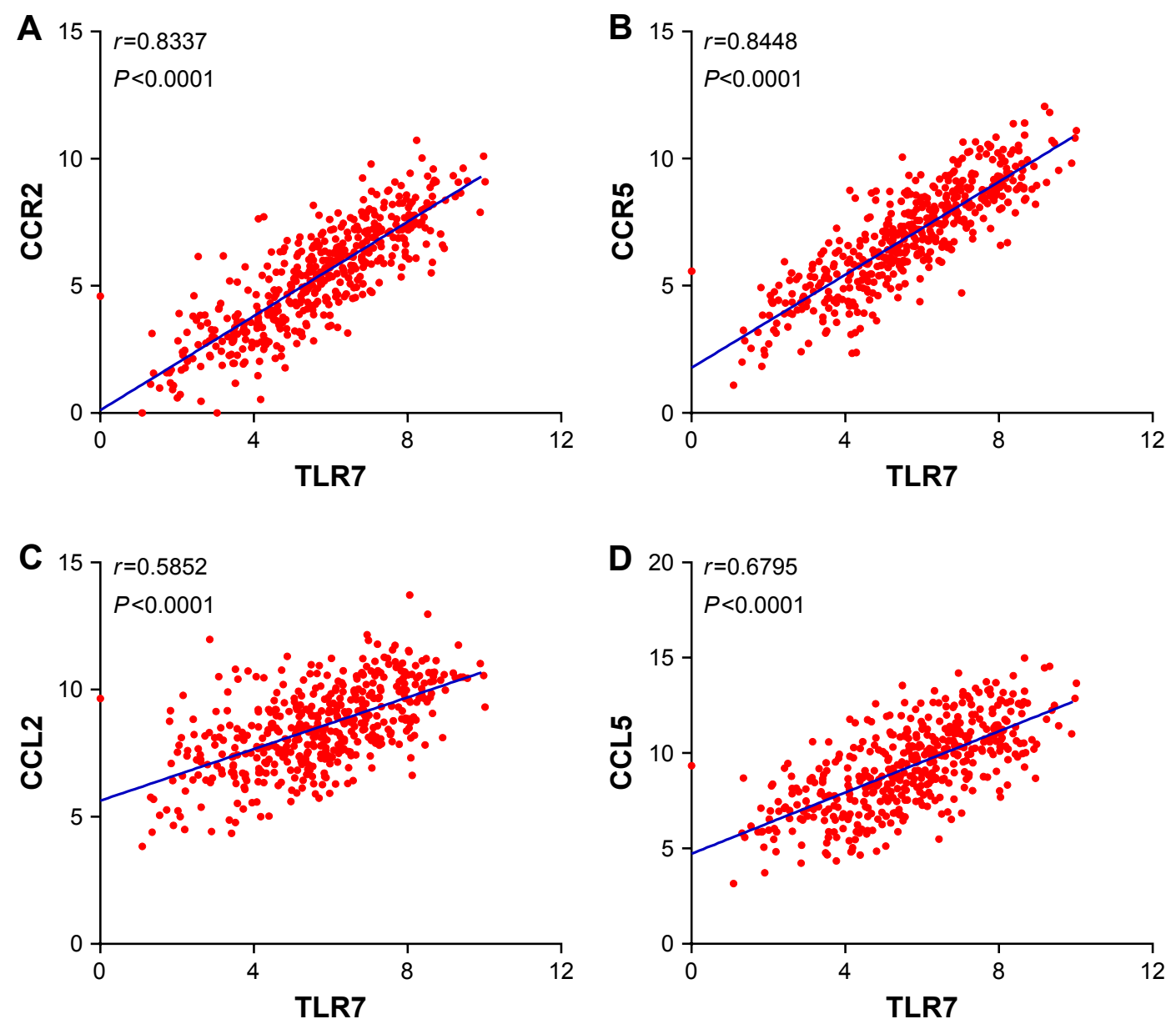

Figure 3 Correlation analysis between expressions of TLR7 and chemokines and their receptors. Correlation analysis between (A) TLR7 and CCR2, (B) TLR7 and CCR5, (C) TLR7 and CCL2, and (D) TLR7 and CCL5.

Note: $r$, Pearson's correlation coefficient.

Abbreviation: TLR, Toll-like receptor.

$\mathrm{CD}^{+} \mathrm{T}$ cells are an important prognostic factor for patients with a variety of cancer types. ${ }^{15}$ An increased presence of $\mathrm{CD}^{+} \mathrm{T}$ cells at the tumor site is a key to obtaining a therapeutic response using immunotherapy (eg, PD-1/PD-L1 antibodies). ${ }^{24}$ In the present study, we demonstrated a strong correlation between the expression levels of TLR7/8 and CD8A. Moreover, we found that expression levels of TLR7/8 were associated with that of $\mathrm{CD}^{+} \mathrm{T}$ cell-specific markers, including perforin, granzyme B, and IFNg. Taken together, these data suggest that TLR7/8 expression is closely associated with $\mathrm{CD}^{+} \mathrm{T}$-cell immunity. Interestingly, we found that the expression of those genes that had a strong positive correlation with TLR7/8 expression $(r>0.5)$ also predicted better patient outcomes. These genes included CCR2, CCR5, CD8A, IFNG, GZMB, and PRF1. These results further validate the correlation between TLR7/8 expression and DC/T-cell infiltration and trafficking in melanoma. Ultimately, these findings may provide new insights for the development of novel immunotherapies to treat melanoma and other types of cancer.

We recognize that there exist several limitations to our study. First, our analysis was primarily performed using an open public database with a large cohort of patients and RNAseq gene data. The gene expression data we collected were for tumor tissues, which contained not just tumor cells but also stromal cells and immune cells. The contribution of each cell type remains unclear and requires further analysis, such as immunohistochemical analysis of TLR protein expression. Second, while our correlation analysis provided important results, functional experiments are required to further validate them.

\section{Conclusion}

We report that TLR7/8 expression represents an independent prognostic factor in patients with melanoma. Our data demonstrate that expression levels of TLR7/8 highly correlated 

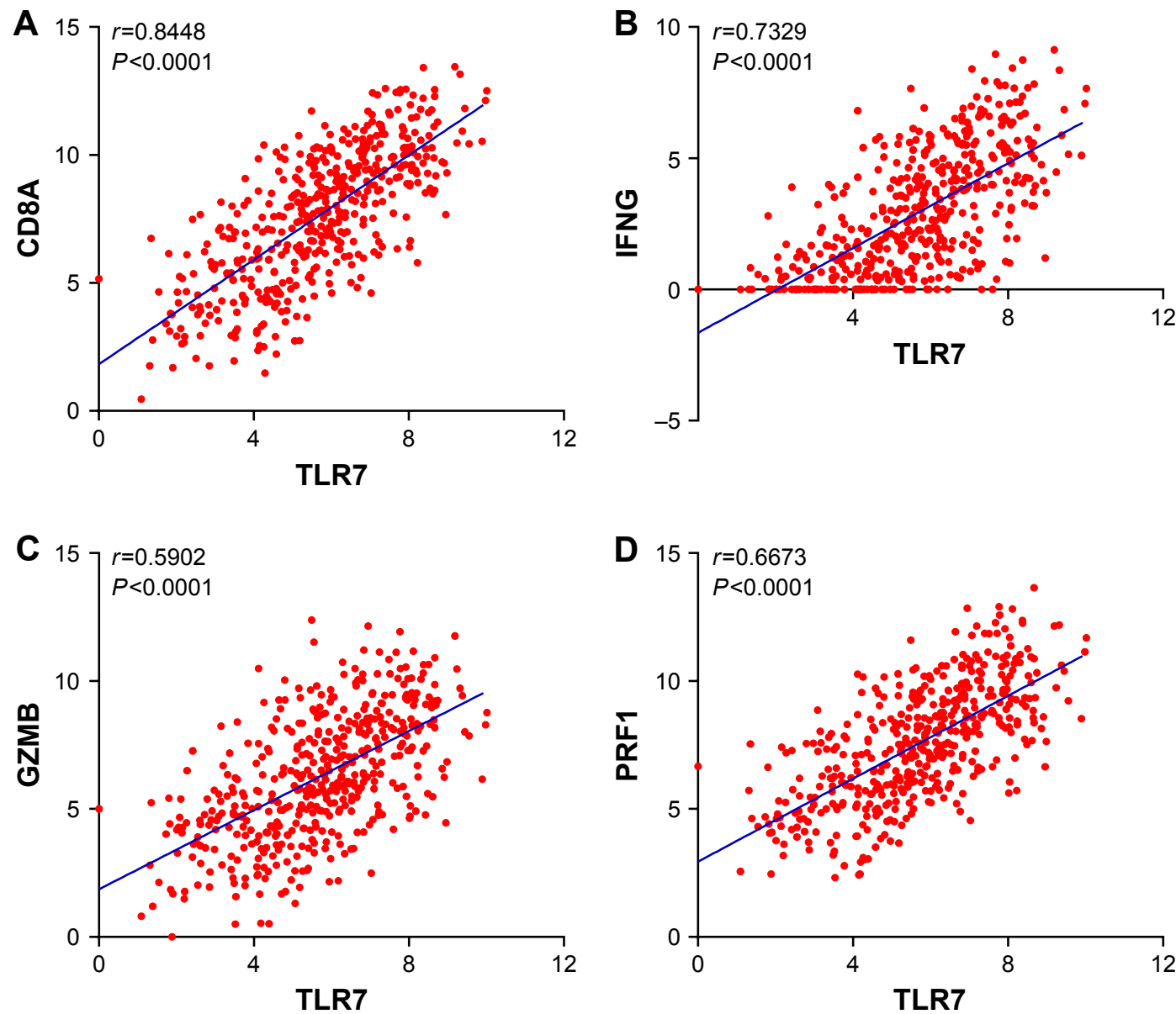

Figure 4 Correlation analysis between expression levels of TLR7 and CD8 ${ }^{+}$cytotoxic T-cell markers. Correlation analysis between (A) TLR7 and CD8A, (B) TLR7 and IFNG, (C) TLR7 and GZMB, and (D) TLR7 and PRFI.

Note: $r$, Pearson's correlation coefficient.

Abbreviations: TLR, Toll-like receptor; IFNG, interferon gamma.

with that of chemokines and their receptors. We also show a strong association between TLR7/8 expression and CD8 ${ }^{+}$ T-cell immunity. Our findings not only provide an important reference for the clinical prognosis of melanoma but also present a new therapeutic modality for the development of melanoma immunotherapy.

\section{Acknowledgment}

This work was supported by the Zhejiang Provincial Medical and Health Project (2016KYA163). The funders had no role in study design, data collection, and analysis; decision to publish this article; or in the preparation of the manuscript.

\section{Disclosure}

The authors report no conflicts of interest in this work.

\section{References}

1. Burns EM, Yusuf N. Toll-like receptors and skin cancer. Front Immunol. 2014;5:135.
2. Akira S, Hemmi H. Recognition of pathogen-associated molecular patterns by TLR family. Immunol Lett. 2003;85(2):85-95.

3. Song PI, Park YM, Abraham T, et al. Human keratinocytes express functional CD14 and toll-like receptor 4. J Invest Dermat. 2002;119(2): 424-432.

4. Bauer S, Pigisch S, Hangel D, Kaufmann A, Hamm S. Recognition of nucleic acid and nucleic acid analogs by Toll-like receptors 7,8 and 9 . Immunobiology. 2008;213(3-4):315-328.

5. Santoni M, Andrikou K, Sotte V, et al. Toll like receptors and pancreatic diseases: from a pathogenetic mechanism to a therapeutic target. Cancer Treat Rev. 2015;41(7):569-576.

6. Ochi A, Graffeo CS, Zambirinis CP, et al. Toll-like receptor 7 regulates pancreatic carcinogenesis in mice and humans. J Clin Invest. 2012; 122(11):4118-4129.

7. Aspord C, Tramcourt L, Leloup C, et al. Imiquimod inhibits melanoma development by promoting $\mathrm{pDC}$ cytotoxic functions and impeding tumor vascularization. J Invest Dermatol. 2014;134(10):2551-2561.

8. Craft N, Bruhn KW, Nguyen BD, et al. The TLR7 agonist imiquimod enhances the anti-melanoma effects of a recombinant Listeria monocytogenes vaccine. J Immunol. 2005;175(3):1983-1990.

9. Goldinger SM, Dummer R, Baumgaertner P, Mihic-Probst SM, Schwarz SM, Hammann-Haenni SM. Nano-particle vaccination combined with TLR-7 and -9 ligands triggers memory and effector CD8(+) T-cell responses in melanoma patients. Eur J Immunol. 2012;42(11): 3049-3061. 
10. Aranda F, Llopiz D, Diaz-Valdes N, et al. Adjuvant combination and antigen targeting as a strategy to induce polyfunctional and high-avidity T-cell responses against poorly immunogenic tumors. Cancer Res. 2011; 71(9):3214-3224.

11. Drobits B, Holcmann M, Amberg N, et al. Imiquimod clears tumors in mice independent of adaptive immunity by converting pDCs into tumor-killing effector cells. J Clin Invest. 2012;122(2):575-585.

12. Eiro N, Ovies C, Fernandez-Garcia B, et al. Expression of TLR3, 4, 7 and 9 in cutaneous malignant melanoma: relationship with clinicopathological characteristics and prognosis. Arch Dermatol Res. 2013; 305(1):59-67.

13. Goldman M, Craft B, Swatloski T, et al. The UCSC Cancer Genomics Browser: update 2013. Nucleic Acids Res. 2013;41(Database issue): D949-D954.

14. Bogunovic D, O’Neill DW, Belitskaya-Levy I, et al. Immune profile and mitotic index of metastatic melanoma lesions enhance clinical staging in predicting patient survival. Proc Natl Acad Sci U S A. 2009; 106(48):20429-20434.

15. Fridman WH, Pages F, Sautes-Fridman C, Galon J. The immune contexture in human tumours: impact on clinical outcome. Nat Rev Cancer. 2012;12(4):298-306.

16. Dajon M, Iribarren K, Cremer I. Toll-like receptor stimulation in cancer: a pro- and anti-tumor double-edged sword. Immunobiology. 2017; 222(1):89-100.
17. Ridnour LA, Cheng RY, Switzer CH, et al. Molecular pathways: tolllike receptors in the tumor microenvironment - poor prognosis or new therapeutic opportunity. Clin Cancer Res. 2013;19(6):1340-1346.

18. Gonzalez-Reyes S, Marin L, Gonzalez L, et al. Study of TLR3, TLR4 and TLR9 in breast carcinomas and their association with metastasis. BMC Cancer. 2010;10:665.

19. Singh M, Khong H, Dai Z, et al. Effective innate and adaptive antimelanoma immunity through localized TLR7/8 activation. J Immunol. 2014;193(9):4722-4731.

20. Chaturvedi A, Pierce SK. How location governs toll-like receptor signaling. Traffic. 2009;10(6):621-628.

21. Smits EL, Ponsaerts P, Berneman ZN, Van Tendeloo VF. The use of TLR7 and TLR8 ligands for the enhancement of cancer immunotherapy. Oncologist. 2008;13(8):859-875.

22. Gauzzi MC, Del Corno M, Gessani S. Dissecting TLR3 signalling in dendritic cells. Immunobiology. 2010;215(9-10):713-723.

23. Schreibelt G, Tel J, Sliepen KH, et al. Toll-like receptor expression and function in human dendritic cell subsets: implications for dendritic cell-based anti-cancer immunotherapy. Cancer Immunol Immunother. 2010;59(10):1573-1582.

24. Arina A, Corrales L, Bronte V. Enhancing T cell therapy by overcoming the immunosuppressive tumor microenvironment. Semin Immunol. 2016;28(1):54-63.
OncoTargets and Therapy

\section{Publish your work in this journal}

OncoTargets and Therapy is an international, peer-reviewed, open access journal focusing on the pathological basis of all cancers, potential targets for therapy and treatment protocols employed to improve the management of cancer patients. The journal also focuses on the impact of management programs and new therapeutic agents and protocols on

\section{Dovepress}

patient perspectives such as quality of life, adherence and satisfaction The manuscript management system is completely online and includes a very quick and fair peer-review system, which is all easy to use. Visit http://www.dovepress.com/testimonials.php to read real quotes from published authors. 